\title{
Fecundidade da manjuba Curimatella lepidura (CURIMATIDAE) na represa de Três Marias, rio São Francisco, MG
}

\author{
[Fecundity of manjuba Curimatella lepidura (CURIMATIDAE) in the Três Marias reservoir, \\ São Francisco River, $M G$ ] \\ D.R. Andrade ${ }^{1}$, M.V. Vidal Junior ${ }^{1}$, H.P. Godinho ${ }^{2}$, W.C.T. Tonini ${ }^{1}$, D. Burket ${ }^{1}$ \\ ${ }^{1}$ LZNA/CCTA - Universidade Estadual do Norte Fluminense \\ Av. Alberto Lamego, 2000 \\ 28013-800 - Campos dos Goytacazes, RJ \\ ${ }^{2}$ Pontifícia Universidade Católica de Minas Gerais - Betim, MG
}

\begin{abstract}
RESUMO
Foram determinados os tamanhos dos ovócitos e as fecundidades residual e relativa ao peso de corpo, ao comprimento padrão, ao peso do ovário e ao peso de corpo do líquido dos ovócitos vitelogênicos, e a fecundidade absoluta de 230 fêmeas de manjubas (Curimatella lepidura) coletadas no reservatório de Três Marias, rio São Francisco, MG durante três anos de coleta. Foi utilizado o método gravimétrico para determinar a quantidade de ovócitos vitelogênicos, que foram separados dos avitelogênicos, por diferença de peso. O diâmetro médio dos ovócitos foi de $711 \pm 44 \mu \mathrm{m}$. O valor médio da fecundidade absoluta foi de 60.994,9 $\pm 27.142,7$ ovócitos por fêmea no estádio 2C de maturação (maduro). A fecundidade média

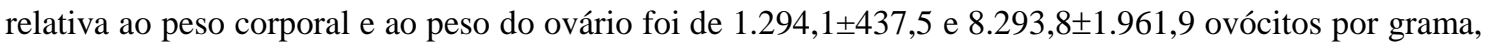
respectivamente. Não houve diferença significativa $(\mathrm{P}<0,05)$ entre as fecundidades absolutas médias nos três anos de pesquisa. Foram encontrados elevados coeficientes de correlação, e os modelos lineares e potenciais foram os mais adequados para expressar as fecundidades absolutas e relativas, ou seja, quanto maior o grau de maturidade, maior a fecundidade dos peixes.
\end{abstract}

Palavras chaves: manjuba, Curimatella, fecundidade, ovócito, reprodução

\begin{abstract}
The sizes of vitellogenic oocytes and fecundity of 230 females of manjubas (Curimatella lepidura) from the Três Marias reservoir, river São Francisco, MG, was determined throughout three years of monthly captures. Gravimetric method was used to determine the quantities of vitellogenic oocytes, which were separated from no vitellogenics by weight difference and using water flow. The average diameter of vitellogenic oocytes was $711 \pm 44 \mu$ m. The mean absolute fecundity was $60994.9 \pm 27142.7$ oocytes per female in stage $2 \mathrm{C}$ of maturation (mature). The average fecundity relative to body weight and ovarian weight was $1294.1 \pm 437.5$ and $8293.8 \pm 1961.9$ oocytes per gram, respectively. There was no significant difference $(P<0.05)$ between fecundity during the three years of research. High correlation coefficients were found, and the linear and potential models were the most appropriate to express the absolute and relative fecundities, i.e., the higher the maturity degree, higher the fecundity.
\end{abstract}

Keywords: Curimatella, fecundity, oocytes, reproduction

\section{INTRODUÇÃO}

A espécie Curimatella lepidura, conhecida vulgarmente como manjuba, piaba, saguiru, branquinha e sairu, pertence à família
Curimatidae, ordem Characiformes. A manjuba é considerada espécie de hábito alimentar detritívoro, sendo um importante peixe na bacia do rio São Francisco, pela abundância e participação na cadeia alimentar. (Pompeu e Godinho, 2003). Segundo Gneri e Angelescu

Recebido em 3 de abril de 2009

Aceito em 26 de novembro de 2010

E-mail: dalcio@uenf.br 
(1951), os peixes detritívoros (ou iliófagos) têm um papel preponderante na bioeconomia do ambiente aquático, podendo atuar como transportador-preparador dos sedimentos de onde vivem. Do ponto de vista puramente econômico, a manjuba pode ser considerada como extratoracumulador, transformando o lodo em proteína corporal disponível para alimentação humana direta. A dieta desta espécie é composta basicamente por algas em decomposição e partículas inorgânicas (Gomes e Verani, 2003). Segundo Sato (1999), esta espécie ocorre com abundância em lagoas marginais do rio São Francisco, raramente ultrapassa $15 \mathrm{~cm}$ de comprimento total e $80 \mathrm{~g}$ de peso corporal.

Os aspectos da pesca, dieta e biologia reprodutiva da manjuba são pouco conhecidos, embora se saiba que a manjuba não necessita realizar migrações reprodutivas, podendo completar seu ciclo de maturação gonadal em ambiente lêntico, como o da represa de Três Marias-MG (Sato e Sampaio, 2005).

A fecundidade está relacionada com a intensidade anual do recrutamento, e o sucesso reprodutivo de um grupo é dependente tanto da quantidade de fêmeas "desovantes" como da fecundidade individual dessas fêmeas (Csirke, 1980). A avaliação da fecundidade representa um subsídio para a estimativa de estoques pesqueiros naturais e para a produção de alevinos em piscicultura (Araújo e Garutti, 2002), e pode fornecer informações sobre a estratégia de vida da espécie, tais como: a definição do estilo de reprodução ou o período de maturação das espécies dulcícolas (Carrassón e Bau, 2003) e marinhas (Laptikhovsky, 2001).

O presente trabalho teve o objetivo de determinar a fecundidade e o tipo de relação entre a fecundidade e parâmetros corporais comprimento padrão, peso corporal, peso das vísceras, peso das gônadas e peso dos ovários de fêmeas de manjuba ( $C$. lepidura) provenientes da represa de Três Marias, rio São Francisco, MG.

\section{MATERIAL E MÉTODOS}

Foram utilizadas 230 fêmeas de manjubas, $C$. lepidura, capturadas na represa de Três Marias, município de Morada Nova, MG (18 35'S e 45 $25^{\prime} \mathrm{W}$ ), durante 35 meses consecutivos (1983-
1985), denominados anos 1, 2 e 3 . A captura foi realizada por meio de redes de emalhar. A transparência da água foi em média $45 \mathrm{~cm}$, e a profundidade onde foram colocadas as redes possuía entre 2 e $3 \mathrm{~m}$. As coletas foram realizadas mensalmente com redes de $200 \mathrm{~m}$ de comprimento e distância entre nós de 5 e $8 \mathrm{~cm}$, as quais foram instaladas por volta das $18 \mathrm{~h}$ e retiradas às $23 \mathrm{~h}$.

Os exemplares capturados foram, após identificação, embalados em sacos plásticos e acondicionados em caixas de isopor contendo gelo e, deste modo, levados para o Laboratório de Ictiologia, do Departamento de Morfologia, ICB, UFMG.

De cada exemplar coletado, foram mensurados o comprimento padrão (CP) e o peso corporal (PC). Foram selecionadas fêmeas com comprimento padrão entre 8,2 e $13,5 \mathrm{~cm}$ e peso entre 17,0 e 73,0g, em possível estado reprodutivo. Posteriormente, foi realizada a exposição das vísceras, mediante a incisão ventral, e retiradas as gônadas para determinação do peso dos ovários com o uso de balança de precisão.

O ovário esquerdo foi destinado à produção de lâminas histológicas e posterior identificação dos estádios de maturação gonadal. O ovário direito de cada exemplar, destinado aos estudos de fecundidade, foi fragmentado e colocado em solução de Gilson modificada, visando à liberação dos ovócitos por meio da digestão do estroma ovariano. A composição da solução de Gilson utilizada foi: $110 \mathrm{~mL}$ de álcool $60 \%$; 880mL de água; $15 \mathrm{~mL}$ de ácido nítrico 60\%; $18 \mathrm{~mL}$ de ácido acético glacial e $20 \mathrm{~g}$ de cloreto de mercúrio. Periodicamente, os ovários foram submetidos à agitação manual para auxiliar a ação da solução até a completa liberação dos ovócitos do estroma, quando, então, foram lavados várias vezes em álcool 70\%.

A massa de ovócitos, assim obtida, foi colocada em uma proveta de $1000 \mathrm{~mL}$ e, por meio de tubo fino de borracha, estabeleceu-se um fluxo de água no sentido fundo-superfície, o qual agitava os ovócitos. O fluxo de água foi regulado de tal forma que os ovócitos menores e mais leves (ovócitos avitelogênicos) fossem arrastados pela corrente ascendente e retirados por transbordamento, restando na proveta, após duas 
ou três horas, apenas os ovócitos vitelogênicos, maiores e mais pesados. Como os ovócitos que transbordavam eram retidos por uma bandeja, a eficiência do processo sempre foi conferida.

A massa obtida de ovócitos vitelogênicos mais pesados, a seguir, passou por secagem em papel de filtro e, posteriormente, em estufa a $40^{\circ} \mathrm{C}$ por duas horas. A massa de ovócitos vitelogênicos secos (até o ponto de retirada externa da água) foi esfriada em temperatura ambiente e, em seguida, pesada em balança de precisão. Dessa massa foram retiradas três subamostras aleatórias e de peso similar. As subamostras foram embrulhadas em papel alumínio, numeradas e estocadas em frascos tampados para posterior contagem e medição do diâmetro dos ovócitos vitelogênicos.

Foi determinada a fecundidade absoluta a partir dos seguintes procedimentos. Os ovócitos de cada subamostra foram depositados em lâminas histológicas contendo ranhuras, onde permaneceram alinhados, em seguida foi realizada sua contagem com o uso de microscópio estereoscópico. Foram medidos 120 ovócitos por ovário do total de 49 fêmeas dentre as coletadas, as quais se encontravam nos estádios intermediário e avançado de maturação gonadal, considerados, respectivamente, como estádios 2B e 2C, os quais foram identificados: estádio 1, em repouso; estádio 2, em maturação; estádio 3, maduro e estádio 4, desovado.

Para a medição dos ovócitos, foi usado o mesmo método de lâminas com ranhuras e microscópio estereoscópico, com ocular micrométrica. A correção para micrômetros foi feita com lâmina micrométrica apropriada. Após determinar-se o número médio de ovócitos e o seu diâmetro em cada subamostra, obteve-se, por regra de três simples, o número total de ovócitos contidos na massa de ovócitos vitelogênicos secos. O valor obtido da contagem realizada dos ovários das 49 fêmeas foi multiplicado por dois para estimar-se o número total de ovócitos vitelogênicos em ambos os ovários do animal.

Como ocorreram modificações histológicas acentuadas ao longo do processo de maturação observadas principalmente no estádio 2, este foi dividido em três subestádios: subestádio 2A, maturação inicial; subestádio 2B, maturação intermediária e subestádio 2C, maturação avançada. A fecundidade absoluta média foi estabelecida em relação aos estádios $2 \mathrm{~A}$ ao $2 \mathrm{C}$, os quais foram identificados a partir de cortes histológicos.

Determinou-se a fecundidade absoluta média do estádio 2C em relação às classes de peso corporal, comprimento padrão e por grama de peso corporal líquido (peso corporal - peso dos ovários) e por peso dos ovários. A esta relação denominou-se fecundidade relativa.

Foi estabelecido o número de ovócitos remanescentes nos ovários no estádio histológico de maturação gonadal 4 (desovado). Este valor, designado como fecundidade residual, foi utilizado tanto na forma de valor absoluto (fecundidade residual absoluta) quanto relativo (fecundidade residual relativa) ao comprimento padrão, ao peso corporal, ao peso corporal líquido e ao peso dos ovários.

Foi realizada, inicialmente, análise de variância e foram utilizados modelos de regressão linear simples e potencial para verificação da existência de relação entre fecundidade absoluta e o comprimento padrão, peso corporal, peso corporal líquido e peso dos ovários.

Para avaliação da ordem de importância da associação entre fecundidade absoluta no estádio 2C de maturação gonadal (maturação avançada) e os parâmetros estudados, considerando as influências mútuas, foi realizada regressão linear múltipla entre eles. A partir de um modelo completo (fecundidade absoluta em função do comprimento padrão, do peso corporal, do peso corporal líquido e do peso dos ovários), as variáveis foram retiradas uma a uma, com base na significância do coeficiente de regressão (procedimento backward). Para contornar o problema de colinearidades, recorreu-se à técnica de componentes principais. Além das regressões calculadas para cada um dos três anos de estudo, também foi realizada regressão global, considerando-se os três anos como período único. A escolha dos modelos linear e potencial foi realizada de acordo com o maior coeficiente de determinação e o melhor ajuste à descrição dos fenômenos estudados.

Foi realizado teste de significância, ano a ano, entre as declividades ("b”) das regressões, pelo teste $\mathrm{t}$, com $5 \%$ de probabilidade, e foram 
determinados as médias, os desvios-padrão, as amplitudes e o coeficiente de variação para as diferentes fecundidades. A fecundidade absoluta do estádio 2C em relação às classes de peso corporal, ao comprimento padrão e ao peso dos ovários foi estabelecida adotando-se cinco classes com idênticos intervalos para o aspecto global do período de estudo. Foi realizada a análise estatística do diâmetro dos ovócitos nos estádios de maturação gonadal 2B, 2C e 4 nos anos estudados, por meio de teste t com $5 \%$ de probabilidade.

\section{RESULTADOS E DISCUSSÃO}

A fecundidade absoluta média das fêmeas de $C$. lepidura, nos diferentes estádios, aumentou conforme o progresso da maturação gonadal, do estádio 2A (maturação inicial) ao estádio 2C (maturação avançada), atingindo o valor máximo neste último estádio (Tab. 1). Não foram encontradas diferenças entre fecundidades absolutas médias nos diferentes anos de estudo. O valor médio da fecundidade absoluta nas fêmeas de $C$. lepidura estudadas foi de
$60.995 \pm 27.143$ ovócitos por indivíduo no estádio de maturação 2C, tendo sido encontrado o valor máximo de 151.214 ovócitos nas fêmeas de maior porte.

Os maiores valores da fecundidade relativa média ao comprimento padrão, ao peso corporal, ao peso corporal líquido e ao peso dos ovários, no estádio de maturação avançada, foram observados no ano de 1985, e os menores, em 1984, com exceção da fecundidade relativa ao peso dos ovários, cujo menor valor ocorreu em 1983 (Tab. 2). O valor médio da fecundidade relativa ao peso dos ovários de C. lepidura no período de estudo foi de 8293,8 ovócitos por grama de ovário. As diferenças entre estes valores, em sua maioria, não foram significativas, exceto em relação ao comprimento padrão (2,334; $\mathrm{P}<0,01)$, ao peso corporal $(3,154$; $\mathrm{P}<0,05)$ e ao peso corporal líquido $(3,374$; $\mathrm{P}<0,05)$ entre os anos de 1983 - 1984 e em relação ao peso dos ovários $(2,289 ; \mathrm{P}<0,05)$ de 1983 - 1985.

Tabela 1. Fecundidade absoluta média (FAM) com suas respectivas amplitudes e coeficientes de variação (CV) de manjubas C. lepidura na represa de Três Marias, em diferentes estádios de maturação gonadal (EM)

\begin{tabular}{llllll} 
& & & \multicolumn{2}{c}{ Amplitude } & \\
\cline { 4 - 5 } EM & $\mathrm{N}$ & FAM & Min. & Máx & CV(\%) \\
\hline 2A & 8 & 6693,4 & 1008,1 & 18310,6 & 78,6 \\
2B & 32 & 20567,9 & 5757,6 & 42086,1 & 50,4 \\
2C & 190 & 60994,9 & 9933,6 & 151214,8 & 44,5 \\
total & 230 & 29418,7 & 1008,1 & 151214,8 & \\
\hline
\end{tabular}

N: número de animaius analisados; 2A: maturação inicial; 2B: intermediário; 2C: avançado.

Tabela 2. Fecundidade relativa média (e desvio-padrão) em relação ao comprimento padrão (CP; ovócitos. $\mathrm{cm}^{-1}$ ), peso corporal (PC; ovócitos. $\mathrm{g}^{-1}$ ), peso líquido (PL; ovócitos. $\mathrm{g}^{-1}$ ) e peso dos ovários (PO; ovócitos. $\left.\mathrm{g}^{-1}\right)$ de manjubas $C$. lepidura na represa de Três Marias

\begin{tabular}{llllll} 
Ano & $\mathrm{N}$ & $\mathrm{CP}$ & $\mathrm{PC}$ & $\mathrm{PL}$ & $\mathrm{PO}$ \\
\hline 1983 & 30 & $4931,2 \pm 2100,6$ & $1205,5 \pm 499,6$ & $1508,6 \pm 724,5$ & $6810,6 \pm 1073,4$ \\
1984 & 102 & $5014,4 \pm 2070,8$ & $1290,7 \pm 441,4$ & $1560,8 \pm 581,8$ & $7901,5 \pm 1863,7$ \\
1985 & 58 & $4725,2 \pm 1534,4$ & $1150,8 \pm 265,9$ & $1332,5 \pm 340,4$ & $7943,6 \pm 1210,1$ \\
total & 190 & $5146,7 \pm 2061,4$ & $1294,1 \pm 437,5$ & $1556,8 \pm 583,2$ & $8293,8 \pm 1961,9$ \\
\hline
\end{tabular}

$\mathrm{N}$ : número de animais analisados.

As equações de regressão linear e o potencial de fecundidade absoluta em função do comprimento padrão, peso corporal, peso líquido e peso dos ovários das manjubas são apresentados na Tab. 3. O modelo potencial foi mais adequado para a avaliação da relação da fecundidade absoluta entre os parâmetros avaliados, sendo que o maior valor de $r^{2}$ nesse tipo de equação ocorreu entre em relação ao peso dos ovários, relação esta, que apresentou também o maior coeficiente de correlação. 
Tabela 3. Parâmetros relativos à regressão linear e ao potencial de fecundidade absoluta em função do comprimento padrão (CP), peso corporal (PC), peso líquido (PL) e peso dos ovários (PO) no estádio avançado de maturação gonadal de 190 manjubas C. lepidura na represa de Três Marias

\begin{tabular}{llllll}
\hline Parâmetro & Modelo & A & B & $\mathrm{r}^{2}$ & $\mathrm{R}$ \\
\hline CP (cm) & Linear $(\mathrm{y}=\mathrm{a}+\mathrm{bx})$ & $-171625,0$ & $19803,2^{*}$ & $0,38^{*}$ & 0,62 \\
& Potencial $\left(\mathrm{y}=\mathrm{ax}{ }^{\mathrm{b}}\right)$ & 2,1086 & $4,1320^{*}$ & $0,42^{*}$ & 0,65 \\
PC (g) & Linear $(\mathrm{y}=\mathrm{a}+\mathrm{bx})$ & $-26821,1$ & $1895,33^{*}$ & $0,43^{*}$ & 0,66 \\
& Potencial $\left(\mathrm{y}=\mathrm{ax}{ }^{\mathrm{b}}\right)$ & 180,80 & $1,4989^{*}$ & $0,46^{*}$ & 0,68 \\
PL (g) & Linear $(\mathrm{y}=\mathrm{a}+\mathrm{bx})$ & $-6539,9$ & $1734,33^{*}$ & $0,26 *$ & 0,51 \\
& Potencial $\left(\mathrm{y}=\mathrm{ax}{ }^{\mathrm{b}}\right)$ & 657,81 & $1,2155^{*}$ & $0,29 *$ & 0,54 \\
PO (g) & Linear $(\mathrm{y}=\mathrm{a}+\mathrm{bx})$ & $-3788,3$ & $8865,78^{*}$ & $0,71^{*}$ & 0,84 \\
& Potencial $\left(\mathrm{y}=\mathrm{ax}{ }^{\mathrm{b}}\right)$ & 7298,1 & $1,0514^{*}$ & $0,74^{*}$ & 0,86 \\
\hline
\end{tabular}

A: intercepto; b: coeficiente de regressão; $\mathrm{r}^{2}$ : coeficiente de determinação; R: coeficiente de correlação;

*: significativo a $1 \%$.

Todos os parâmetros mostraram estar relacionados com a fecundidade absoluta, pois as declividades "b" e os coeficientes de determinação "r" foram sempre significativos a
1\%. No entanto, o peso dos ovários teve o maior coeficiente de correlação com a fecundidade (Tab. 4).

Tabela 4. Regressão múltipla de fecundidade absoluta em função do comprimento padrão (CP), peso corporal (PC), peso líquido (PL) e peso dos ovários (PO) no estádio avançado de maturação gonadal de 190 manjubas C. lepidura na represa de Três Marias

\begin{tabular}{llll}
\hline Variáveis & Completo & Menos PL & Menos PL, PC \\
\hline Intercepto & $-86624,3$ & $-86623,9$ & $-71153,5$ \\
PL & $-468,396$ & - & - \\
PC & 218,2886 & $-250,11$ & - \\
CP & 8683,363 & 8683,33 & 6499,21 \\
PO & 7347,093 & 7815,49 & 7623,53 \\
R2 & 0,741866 & 0,741866 & 0,740824 \\
CV & 22,678 & 22,616 & 22,602 \\
F para redução & - & $0,00005 N S$ & $0,050453 N S$ \\
\hline
\end{tabular}

Considerando-se apenas os anos 2 (1984) e 3 (1985) do estudo (período com maior número de fêmeas maduras), a fecundidade residual absoluta média foi mais elevada, com 14.402 ovócitos fecundados por grama de gônada, com coeficiente de variação de 69,5\% (Tab. 5). A fecundidade residual absoluta apresentou correlação $(\mathrm{P}<0,01)$ com o peso dos ovários, mas não houve correlação com o comprimento destes.
As variações anuais da fecundidade residual relativa média (Tab. 6) em função do comprimento padrão, peso corporal e peso corporal líquido não foram acentuadas $\left(\mathrm{r}^{2}=\right.$ 0,121, 0,143, 0,061, respectivamente). Em relação ao peso do ovário o $r^{2}$ foi igual a 0,845 nos modelos lineares, ocorrendo o mesmo comportamento para os modelos potenciais testados.

Tabela 5. Fecundidade residual absoluta média (FRAM) no estádio desovado (4) de maturação gonadal de fêmeas da manjuba C. lepidura na represa de Três Marias, durante o segundo e terceiro anos (1984-1985) de estudo

\begin{tabular}{llllll}
\hline & & & \multicolumn{3}{c}{ Amplitude } \\
\cline { 4 - 5 } Ano & $\mathrm{N}$ & FRAM & Mínima & Máxima & CV(\%) \\
\hline 1984 & 15 & 14356,7 & 1450,7 & 35795,1 & 75,5 \\
1985 & 8 & 14486,4 & 3219,2 & 28838,2 & 62,3 \\
Total & 23 & 14401,8 & 1450,7 & 35795,1 & 69,5 \\
\hline
\end{tabular}

$\mathrm{N}$ : número de animais analisados. 


\section{Andrade et al.}

Tabela 6. Fecundidade residual relativa média em relação ao comprimento padrão (CP; ovócitos. $\left.\mathrm{cm}^{-1}\right)$, ao peso corporal (PC; ovócitos. $\mathrm{g}^{-1}$ ), ao peso líquido (PL; ovócitos. $\mathrm{g}^{-1}$ ) e ao peso dos ovários (PO; ovócitos. $\mathrm{g}^{-1}$ ) de fêmeas da manjuba $C$. lepidura na represa de Três Marias, durante o segundo e terceiro anos (1984-1985) de estudo no estádio desovado (4) de maturação gonadal

\begin{tabular}{llrrrr}
\hline Ano & $\mathrm{N}$ & $\mathrm{CP} \pm \mathrm{DP}$ & $\mathrm{PC} \pm \mathrm{DP}$ & $\mathrm{PL} \pm \mathrm{DP}$ & $\mathrm{PO} \pm \mathrm{DP}$ \\
\hline 1984 & 15 & $1159,8 \pm 849,9$ & $274,4 \pm 197,1$ & $297,8 \pm 226,8$ & $4674,7 \pm 1583,1$ \\
1985 & 8 & $1238,4 \pm 763,1$ & $320,5 \pm 202,2$ & $244,6 \pm 222,4$ & $4992,4 \pm 1802,3$ \\
Total & 23 & $1187,1 \pm 804,0$ & $290,5 \pm 195,8$ & $314,1 \pm 221,3$ & $4185,2 \pm 1628,6$
\end{tabular}

N: número de animais analisados; DP: desvio-padrão.

O diâmetro dos ovócitos vitelogênicos, no estádio 2C (maturação avançada), manteve-se estatisticamente inalterado e com média geral de $711 \pm 44 \mu \mathrm{m}$, durante o período de estudo. O diâmetro dos ovócitos remanescentes do estádio 4 (desovado) não diferiu significativamente dos ovócitos 2C. Segundo Mazzoni et al. (2002), não há correlação entre diâmetro dos ovócitos e fecundidade e nem com o tamanho dos peixes, em estudos realizados com Characiidae.

Existem diversos fatores que podem interferir na fecundidade e produção ovocitária de uma espécie de peixe e, assim, acarretar modificações na quantidade de ovócitos viáveis, entre eles: idade (Alp et al., 2003), nutrição (Chong et al., 2004), diferenças entre populações (Duponchelle et al., 2000) e aspectos ambientais como temperatura, variações de $\mathrm{pH}$ e salinidade (Kraus et al., 2000; Campos-Mendoza et al., 2004).

Dentre as espécies encontradas no rio São Francisco, a manjuba $C$. lepidura é uma das que apresentam maior quantidade de ovócitos por grama de ovário (Sato, 1999). A produtividade de ovócitos geral obtida neste estudo encontra-se bem próxima dos valores das fecundidades apresentadas por outras espécies de caracídeos, sendo mais elevada que a encontrada em Astyanax fasciatus e Astyanax bimaculatus (Vazzoler, 1996), Serrapinnus piaba (Silvano et al., 2003) e mais baixa que a verificada em Galeocharax knerii e Salminus maxillosus (Vazzoler, 1996). No entanto, a fecundidade dos caracídeos varia muito em virtude do tamanho corporal da espécie, pois peixes menores têm menor capacidade de armazenar ovócitos (Bagenal e Braun, 1978). Assim, a melhor forma de se comparar a fecundidade entre espécies de um mesmo gênero é utilizando a fecundidade relativa.
De forma geral, é encontrada uma acentuada correlação entre fecundidade e comprimento corporal (Kume et al., 2000). Pode haver situações como em Clupea harengus, em que o peso corporal foi considerado como o melhor indicador de fecundidade mesmo sendo encontrada alta correlação entre fecundidade e comprimento (Óskarsson et al., 2002). Em tilápia-do-nilo, há correlação também entre fecundidade e idade dos peixes, em que os mais velhos apresentam fecundidade relativa menor do que animais mais jovens (Desprez et al., 2008).

De acordo com Bagenal e Braun (1978) o comprimento é o parâmetro mais adequado para estimar a correlação com a fecundidade, isso acontece, pois o peixe não aumenta significativamente de tamanho enquanto desenvolve suas gônadas, sendo esta relação explicada na forma de uma equação potencial. Por outro lado, exemplos como os de Dadzie et al. (2000) e Stequert et al. (2003) permitem descrever a fecundidade de forma linear tanto para o comprimento quanto para o peso corporal.

Godinho et al. (1977) descreveram baixos coeficientes de correlação entre fecundidade e comprimento ou peso, os quais são indicativos de grande variação na fecundidade para peixes de um dado comprimento e peso, refletindo variações nas condições alimentares. Essa observação confirma os baixos valores de correlações entre os parâmetros estudados no caso de C. lepidura, avaliados durante os três anos de estudo. A fecundidade relativa pode ser estabelecida por meio de relações entre comprimento total, peso total e fecundidades, expressas por equações do tipo potencial (Vazzoler, 1996). A determinação da fecundidade relativa apresenta algumas dificuldades, principalmente quando esta é relacionada ao peso. Isso acontece, pois, em 
muitos peixes, o número de ovócitos não muda durante o período de reprodução, enquanto o peso da gônada aumenta em virtude de acréscimo de água ou matéria orgânica derivada da vitelogenina (Bagenal e Braun, 1978). Sendo um fator correlacionado com o peso e o bemestar dos animais, a fecundidade pode ser influenciada também pela densidade de estoque, qualidade de água e até mesmo pela qualidade do alimento fornecido (Tsadik e Bart, 2007).

A fecundidade relativa de $C$. lepidura neste estudo foi mais elevada que o valor médio obtido por Sato (1999) utilizando a hipofisação. Assim como no presente estudo, Stequert et al., (2003) obtiveram maiores valores para correlações entre fecundidade e os parâmetros tomados do próprio ovário. No caso das manjubas, observa-se que a fecundidade relativa média cresce potencialmente 1,05 vez a cada grama de ovário, apresentando uma média de $8294 \pm 1962$ ovócitos a cada grama de ovário.

A relação entre aumento da fecundidade e peso é geralmente apresentada como proporcional e cresce uniformemente (Sokolowska e Skóra, 2001). No presente caso, pode ser descrita curvilinearmente pelo peso dos ovários, sugerindo que este seja um bom índice para que se possa determinar a fecundidade da espécie.

\section{CONCLUSÕES}

A fecundidade absoluta anual desta espécie mostrou-se praticamente constante durante vários anos e aumentou progressivamente em relação ao comprimento padrão, peso corporal e peso dos ovários. Em função do pequeno porte e da alta quantidade de ovos produzidos, pode-se atribuir que $C$. lepidura é uma espécie adequada a reprodução em cativeiro para diversos fins.

\section{REFERÊNCIAS BIBLIOGRÁFICAS}

ALP, A.; KARA, C.; BÜYÜKÇAPAR, H.M. Reproductive biology of brown trout, Salmo trutta macrostigma Dumeril 1858, in a tributary of the Ceyhan River which flows into the eastern Mediterranean Sea. J. Appl. Ichthyol., v.19, p.346-351, 2003.
ARAÚJO, R.B.; GARUTTI, V. Biologia reprodutiva de Aspidoras fuscoguttatus (Siluriformes, Callichthyidae) em riacho de cabeceira da bacia do alto rio Paraná. Iheringia, Série Zoológica., v.92, p.89-98, 2002.

BAGENAL, T.B.; BRAUN, E. Eggs and early life history. In: BAGENAL, T.B (Ed). Methods for assessment of fish production in freshwaters. 3.ed. Oxford: Blackwell, 1978. p.154-190.

CAMPOS-MENDOZA, A.; MCANDREW, B.J.; COWARD, K. et al. Reproductive response of Nile tilapia (Oreochromis niloticus) to photoperiodic manipulation; effects on spawning periodicity, fecundity and egg size. Aquaculture, v.231, 299-314, 2004.

CARRASSÓN, M.; BAU, M. Reproduction and gonady histology of Aidablennius sphinx (Piscis, Blenniidae) of the Catalan sea (Northwestern Mediterranean). Sci. Marina, v.67, p.461-469, 2003.

CHONG, A.S.C.; ISHAK, S.D.; OSMAN, Z. et al. Effect of dietary protein level on the reproductive performance of female swordtails Xiphophorus helleri (Poeciliidae). Aquaculture, v.234, p.381-392, 2004.

CSIRKE, J. Introducción a la dinámica de poblaciones de peces. Roma: FAO, 1980. p.1-82. (Doc. Pesca, FAO/192).

DADZIE, S.; ABOU-SEEDO, F.; ALSHALLAL, T. Reproductive biology of the silver pomfret, Pampus argenteus (Euphrasen), in Kuwait waters. J. Appl. Ichthyol., v.16, p.247-253, 2000.

DESPREZ, D.; BOSC, P.; BAROILLER, J.F. et al. Variability in reproductive performance of sex-reversed tilapia Oreochromis aureus. Aquaculture, v.277, p.73-77, 2008.

DUPONCHELLE, F.; CECCHI, P.; CORBIN, D. et al. Variations in fecundity and egg size of female Nile tilapia, Oreochromis niloticus, from man-made lakes of Côte d'Ivoire. Environ. Biol. Fishes, v.57, p.155-170, 2000.

GNERI, F.S.; ANGELESCU, V. La nutricion de los peces iliofagos em relacion com el metabolismo general del ambiente acuatico. Ver. Inst. Invest. Argent. Cienc. Nat. Cienc. Zool., v.2, p.1-44, 1951. 
GODINHO, H.M; BASILE-MARTINS, M.A.; FENERICH, N.A. et al. Fecundidade e tipo de desova do mandi Pimelodus maculatus Lacepédè, 1803 (Pisces Siluroidei). Rev. Bras. Biol., v.34, p.581-583, 1977.

KRAUS, G.; MÜLLER, A.; TRELLA, K. et al. Fecundity of Baltic cod: temporal and spatial variation. J. Fish Biol., v.56, p.1327-1341, 2000.

KUME, G.; YAMAGUCHI, A.; AOKI, I. et al. Reproductive biology of cardinalfish Apogon lineatus in Tokyo Bay, Japan. Fish. Sci., v.66, p.947-954, 2000.

LAPTIKHOVSKY. V. First date of ovary maturation and fecundity in the squid family Histioteuthidae. Sci. Marina, v.65, p.127-129, 2001.

MAZZONI, R.; CARAMASCHI, E.P.; FENERICH-VERANI, N. Reproductive biology of a Characidiinae (Osteichthyes, Characidae) from the Ubatiba River, Maricá - RJ. Braz. J. Biol., v.62, p.487-494, 2002.

ÓSKARSSON, G.J.; KJESBU, O.S.; SLOTTE, A. Predictions of realized fecundity and spawning time in Norwegian spring-spawning herring (Clupea harengus). Journal of Sea Research, v.48, p.59- 79, 2002.

POMPEU, P.S.; GODINHO, H.P. Effects of extended absence of flooding on the fish assemblages of three floodplain lagoons in the middle São Francisco River, Brazil. Neotrop. Ichthyol., v.4, p.427-433, 2006.

SATO, Y. Reprodução de peixes da bacia do rio São Francisco: Indução e caracterização de padrões, 1999. 179f. Tese (Doutorado) Universidade Federal de São Carlos, São Carlos, SP.
SATO, Y.; SAMPAIO, E.V. A ictiofauna na região do alto São Francisco, com ênfase no reservatório de Três Marias, Minas Gerais. In: NOGUEIRA, M.G.; HENRY, R.; JORCIN, A. (Eds). Ecologia de reservatórios: impactos potenciais, ações de manejo e sistemas em cascata. São Carlos: Rima, p.251-304.

SILVANO, J.; OLIVEIRA, C.L.C.; FIALHO, C.B. et al. Reproductive period and fecundity of Serrapinnus piaba (Characidae: Cheirodontinae) from the rio Ceará Mirim, Rio Grande do Norte. Braz. Neotrop. Ichthyol., v.1, p.61-66, 2003.

SOKOLOWSKA, E.; SKÓRA, K.E. Fecundity of ninespine stickleback (Pungitius pungitius L., 1758) in the Puck bay. Acta Ichthyol. Piscat., v.31, p.45-60, 2001.

STEQUERT, B.; MENARD, F.; MARCHAL, E. Reproductive biology of Vinciguerria nimbaria in the equatorial waters of the eastern Atlantic Ocean. J. Fish Biol., v.62, p.1116-1136, 2003.

TSADIK, G.G.; BART, A.N. Effects of feeding, stocking density and water-flow rate on fecundity, spawning frequency and egg quality of Nile tilapia, Oreochromis niloticus (L.). Aquaculture, v.272, p.380-388, 2007.

VAZZOLER, E. Biologia da reprodução de peixes teleósteos: teoria e prática. Maringá: EDUEM, 1996.169p. 